\title{
Cerebrospinal Fluid Monoaminergic Metabolites in Wild Papio anubis and P. hamadryas Are Concordant with Taxon-specific Behavioral Ontogeny
}

\author{
Clifford J. Jolly • Jane E. Phillips-Conroy • \\ Jay R. Kaplan • J. John Mann
}

Published online: 20 January 2009

(C) Springer Science + Business Media, LLC 2009

Due to a production error, the author corrections were not incorporated into the published version of the manuscript.

1. Improved versions of Figs. 1 and 2 are printed with their captions:

Fig. 1

Fig. 2

2. Table 5, missing from the published version, is given on this page

Table 5

3. Reference citations in text are corrected by section, with the affected beginning portions of the paragraphs republished under the appropriate section heading, followed by a listing of references to be added to the original published paper.

The online version of the original article can be found at http://dx.doi.org/10.1007/s10764-008-9318-x.

C. J. Jolly $(\bowtie)$

Department of Anthropology, New York University, New York, NY 10003, USA

e-mail: clifford.jolly@nyu.edu

J. E. Phillips-Conroy

Department of Anatomy and Neurobiology, Washington University Medical School, and Department of Anthropology, Washington University,

St. Louis, MO 63130, USA

J. R. Kaplan

Department of Pathology (Comparative Medicine),

Wake Forest University Primate Center, Wake Forest University School of Medicine,

Winston-Salem, NC 27157, USA

\section{J. J. Mann}

Department of Molecular Imaging and Neuropathology, New York State Psychiatric Institute,

and Department of Psychiatry, Columbia University,

New York, NY 10032, USA 

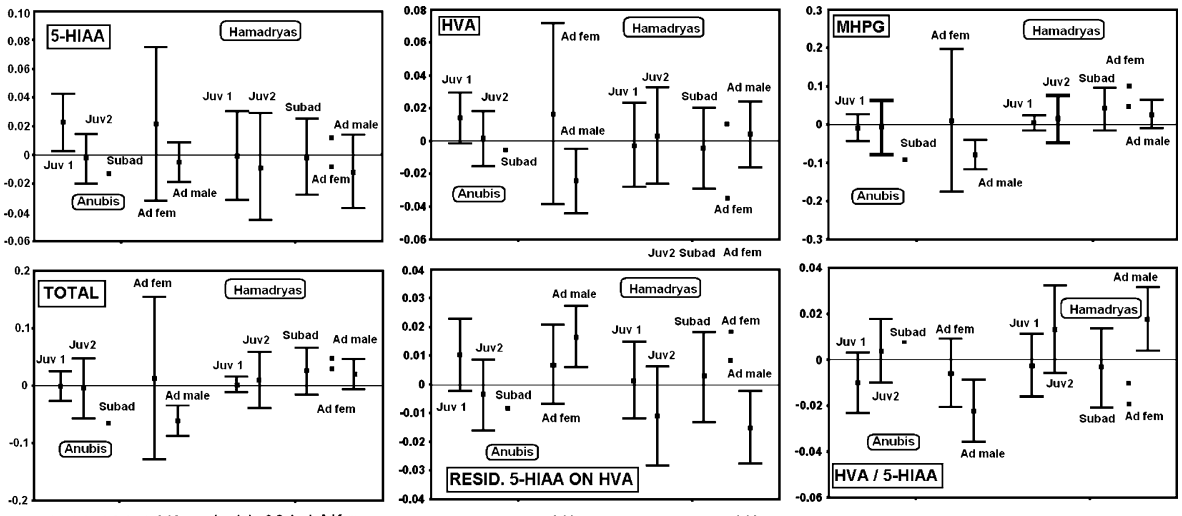

Juv 1 Juv2 Subad Ad tem Juv 1 Juv2 subad Ad tem

Juv 1 Juv2 Subad Ad fem Juv 1 Juve subad Ad fem

Fig. $195 \% \mathrm{CL}$ of mean values of residuals of monoamine variables on estimated age, by age class. Age classes: Juv 1: $\geq 1$ permanent first molar visible; Juv 2: $\geq 1$ permanent second molar visible; Subad: $\geq 1 \mathrm{M} 3$ visible, but dentition incompletely erupted; Ad fem: female with all permanent teeth in full occlusion; Ad male: male with all permanent teeth in full occlusion.
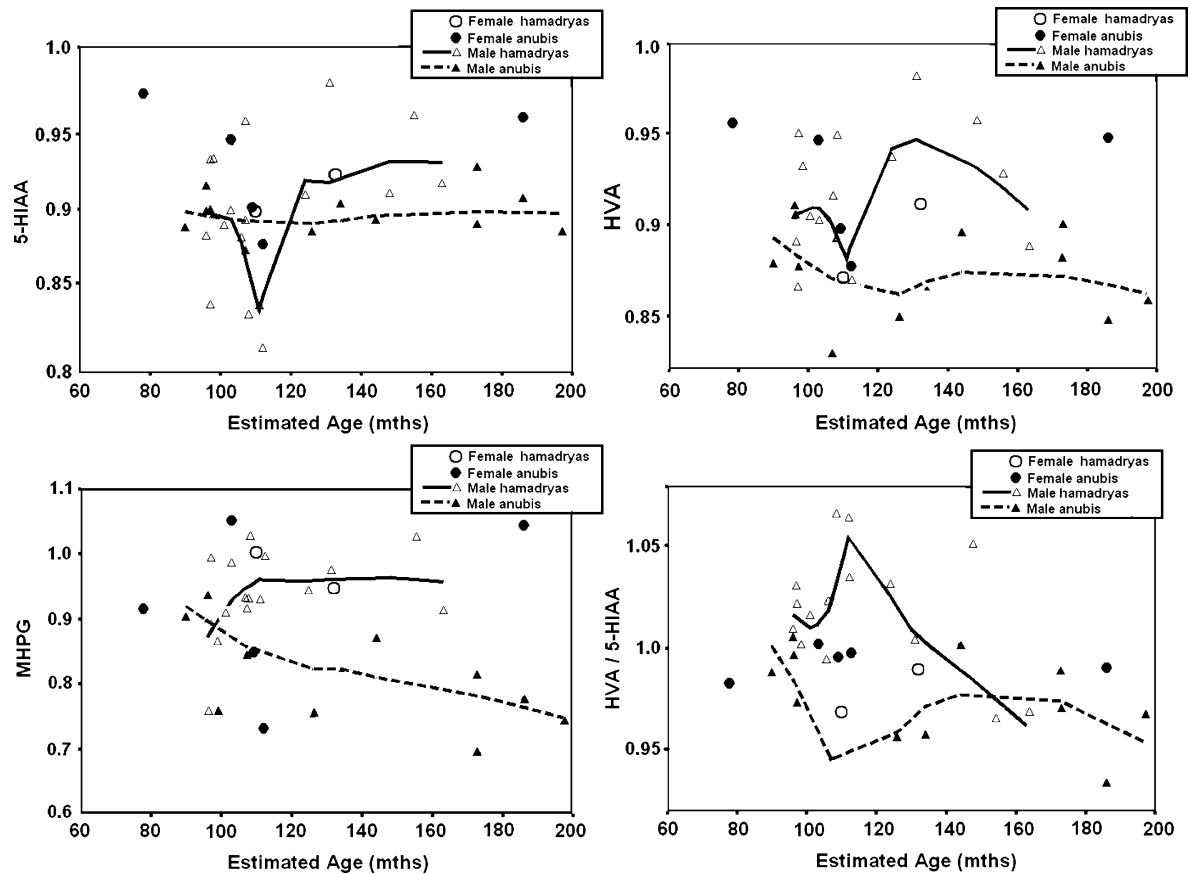

Fig. 2 Trajectories of monoamine variables with age in subadult and adult baboons ( $\geq 1$ M3 visible), by sex and taxon. Lines are LOWESS plots, with 3 iterations, $70 \%$ of points fitted (SPSS). 
Table 5 Mean and SD for monoamine metabolites by dentally-defined age class

\begin{tabular}{|c|c|c|c|c|c|c|c|c|c|c|c|c|c|}
\hline & \multicolumn{2}{|c|}{ 5-HIAA } & \multicolumn{2}{|l|}{ HVA } & \multicolumn{2}{|c|}{ MHPG } & \multicolumn{2}{|c|}{ TOTAL } & \multicolumn{2}{|c|}{ RESID } & \multicolumn{2}{|c|}{ RATIO } & \multirow[t]{2}{*}{$\mathrm{N}$} \\
\hline & Mean & $\mathrm{SD}$ & Mean & $\mathrm{SD}$ & Mean & $\mathrm{SD}$ & Mean & $\mathrm{SD}$ & Mean & $\mathrm{SD}$ & Mean & $\mathrm{SD}$ & \\
\hline \multicolumn{14}{|l|}{ ANUBIS } \\
\hline 1 -No molars & 0.99 & 0.03 & 0.99 & 0.03 & 0.98 & 0.06 & 0.98 & 0.05 & 0.01 & 0.01 & 1.00 & 0.01 & 6 \\
\hline $2-\mathrm{M} 1$ & 0.96 & 0.03 & 0.97 & 0.03 & 0.96 & 0.06 & 0.97 & 0.04 & 0.00 & 0.02 & 1.01 & 0.02 & 13 \\
\hline $3-M 2$ & 0.93 & 0.02 & 0.94 & 0.02 & 0.94 & 0.10 & 0.94 & 0.07 & -0.01 & 0.02 & 1.02 & 0.02 & 10 \\
\hline $4-\mathrm{M} 3$ & 0.90 & & 0.91 & & 0.83 & & 0.86 & & -0.01 & & 1.01 & & 1 \\
\hline 5 -Ad female & 0.93 & 0.04 & 0.92 & 0.04 & 0.92 & 0.14 & 0.92 & 0.10 & 0.01 & 0.01 & 0.99 & 0.01 & 5 \\
\hline 5 -Ad male & 0.90 & 0.02 & 0.87 & 0.03 & 0.81 & 0.08 & 0.83 & 0.06 & 0.02 & 0.02 & 0.97 & 0.02 & 13 \\
\hline \multicolumn{14}{|l|}{ HAMADRYAS } \\
\hline 1 -No molars & 1.00 & 0.04 & & 0.02 & & 0.05 & & 0.03 & & .03 & 00 & 0.03 & 3 \\
\hline $2-\mathrm{M} 1$ & 0.94 & 0.05 & 0.95 & 0.04 & 0.97 & 0.03 & 0.97 & 0.02 & -0.01 & 0.02 & 1.02 & 0.02 & 11 \\
\hline $3-\mathrm{M} 2$ & 0.92 & 0.06 & 0.94 & 0.05 & 0.96 & 0.10 & 0.95 & 0.08 & -0.01 & 0.03 & 1.03 & 0.03 & 1 \\
\hline $4-\mathrm{M} 3$ & 0.92 & 0.03 & 0.92 & 0.03 & 0.97 & 0.07 & 0.95 & 0.05 & 0.00 & 0.02 & 1.00 & 0.02 & 8 \\
\hline 5 -Ad female & 0.91 & 0.02 & 0.89 & 0.03 & 0.97 & 0.04 & 0.95 & 0.02 & 0.02 & 0.01 & 0.98 & 0.01 & 2 \\
\hline 5 -Ad male & 0.90 & 0.05 & 0.91 & 0.03 & 0.94 & 0.06 & 0.93 & 0.04 & -0.01 & 0.03 & 1.02 & 0.03 & 17 \\
\hline
\end{tabular}

\section{Introduction}

The idea that neurotransmitter dynamics are persistent traits of individuals, and have a genetic basis, has been strengthened by the demonstration of significant correlation between behavioral measures and polymorphisms at genetic loci involved in pathways of production and deployment of the monoaminergic neurotransmitters (Emanuele et al. 2007; Klein et al. 2007). Monoaminergic activity is reflected in cerebrospinal fluid (CSF) levels of the respective metabolites: 3-methoxy-4hydroxyphenylglycol (MHPG), the norepinephrine metabolite; 5-hydroxyindoleacetic acid (5-HIAA), the serotonin metabolite; and homovanillic acid (HVA), the dopamine metabolite. For example, by using CSF metabolite concentrations as indicators of transmitter release and turnover, researchers documented, an inverse association between serotonergic activity and aggressiveness in vervets (Cercopithecus sabaeus) and macaques (Macaca sp.) (Botchin et al. 1993; Higley et al. 1992a; Raleigh et al. 1992), and violence and impulsiveness in humans (Asberg et al. 1986; Berman et al. 1997; Brown et al. 1982; Manuck et al. 2005).

Researchers have linked relatively high central serotonergic activity to positive social interaction in human subjects (Knutson et al. 1998) and in male macaques (Botchin et al. 1993; Higley et al. 1992a). Conversely, a low HVA/5-HIAA ratio, i. e., relatively high serotonin activity by this measure, is associated in humans with depression, attempted suicide, and attention-deficit hyperactivity disorder (ADHD)associated behavior in children (Soderstrom et al. 2003). Gene-environment interaction has been demonstrated in macaques (Macaca mulatta) among carriers of a low-expressing serotonin transporter gene promoter allele, wherein peer-reared individuals have lower CSF 5-HIAA levels in adulthood and more pronounced aggressive behavior than those of maternal-reared controls (Suomi 2006).

To primatologists, such associations are of interest because they might represent persistent personality traits of individuals that help to determine both intragroup 
dynamics, e.g., the coexistence of more and less aggressive animals, and alternative life-history strategies, e.g., the readiness of a male rhesus monkey (Macaca mulatta) to leave his natal group before breeding (Howell et al. 2007; Kaplan et al. 1999; Mehlman et al. 1997).

\section{Materials and Methods}

\section{Data Analysis}

Before analysis, we transformed all metabolite values to natural logarithms to normalize their distributions and to reduce variance. Initial examination showed that plots of metabolite concentrations against estimated age are very similar in slope in the 2 taxa, but the intercept for 5-HIAA is consistently higher in hamadryas. Because the period of initial storage at $-20^{\circ} \mathrm{C}$ was shorter for the hamadryas samples, collected in 1997, and because 5-HIAA is reported to decline slowly in concentration under such conditions (Kobayashi et al. 1987; Langlais et al. 2001), we could not exclude the possibility that an artifact was responsible for the higher 5HIAA in all hamadryas. We therefore adopted the conservative approach of expressing metabolite concentrations of juveniles and adults as a fraction of the mean values of infants tested in the same batch. Because batches and taxa are almost entirely coincident, calibrating against infant values by batch also meant that we calibrated all subjects against infants of their own taxon, with the significant exception of the anubis baboon caught in a hamadryas group in 1997. Though the approach prohibits cross-taxonal comparisons of absolute values, the infants provide standards from which to plot the ontogenetic trajectories for each taxon. The overall ranges of calibrated values, especially of 5-HIAA, were much closer in the 2 taxa than the raw values were. Significantly, the anubis baboon trapped in 1997 had raw values that fell far outside the range of its taxon, but its calibrated values situated it as expected, reinforcing our suspicion that a batch-specific storage effect, rather than a specific difference, underlay the distinction in the metabolite-on-age regression. We conducted all subsequent analyses on the log-transformed, calibrated values.

We also computed the ratio of HVA to 5-HIAA. Several studies (Geracioti et al. 1998; Kahn et al. 1993; Lewine et al. 1991; Soderstrom et al. 2003; Vaughn et al. 1988) suggest that the ratio may be a more stable trait of individuals, and hence a more accurate index of dopaminergic activity, than the absolute values of the neurotransmitter metabolites. The method also controls for variance attributable to metabolite transport into and out of the CSF.

\section{Results}

Correlations among Metabolite Concentrations

Table 2 contains monoaminergic metabolite values for each subject. As in the pilot study (Kaplan et al. 1999), linear correlations between pairs of metabolites are generally high and highly significant (Table 3). We obtained similar results when the 
correlation was controlled for total metabolite concentration and in subsets defined by sex or by taxon (Table 4). Within the subsamples, the correlation between 5HIAA and HVA is consistently the strongest, at 0.8-0.9, while those involving MHPG are always lower and less significant statistically.

\section{Trajectories of Maturation}

ANOVA by age-class, taxon, and sex over the whole data set indicated that age-class was the most consistently significant correlate for all metabolites, and the only significant correlate for 5-HIAA and HVA (Table 4). As Kaplan et al. (1999) reported, total CSF monoamines, and all individual metabolite concentrations, correlate negatively with dental maturation in the sample as a whole, and in each subset (Table 3). HVA is consistently the strongest correlate of dental development, against estimated age (Fig. 2).

\section{Discussion}

Our previous work (Kaplan et al. 1999) and information derived from rhesus monkeys (Mehlman et al. 1995; Trefilov et al. 2000), predicted that the anubis male's strong disposition to dispersal might be associated with taxon-specific, lower serotonergic activity, especially in the late-juvenile to early adulthood span, during which first dispersal typically occurs.

In hamadryas, metabolite values averaged over all adult males include a higher than predicted HVA/5-HIAA ratio, to which both metabolites contribute. However, inspection of the LOWESS-plotted trajectory, suggests that this is attributable largely to young adults (ca. 7-10 yr), which are strongly represented in our sample, depressing the mean for the whole adult age class. The HVA/5-HIAA ratio rises to a peak at ca. $9 \mathrm{yr}$, when it starts to decline. Two of the 3 sampled males $>12$ yr have much lower values, overlapping those of comparably aged male anubis. Most young adult males, though close to the adult mean in testicular size and body mass (Phillips-Conroy and Jolly 2004; Jolly and Phillips-Conroy 2003, 2006), are likely to be unattached bachelors or followers of an OMU, without an OMU of their own (Abegglen 1984; Kummer 1968), an interpretation supported by our observations ad hoc at the trapping site.

Another striking difference between adult males of the 2 taxa is in MHPG levels, which in anubis are low, and continue to decline throughout adult life. The effect is presumably related to the age-related loss of noradrenergic neuron cell bodies in the locus coeruleus, but why it should distinguish the 2 taxa in our study is not obvious. Fecal samples from adult males immediately after trapping show significantly lower cortisol levels in male anubis than in hamadryas (Awash National Park Baboon Research Project, unpubl. data), reflecting a response to events preceding the trapping itself. Beehner (2004) showed that fecal cortisol levels in her study population of (hybrid) baboons were more elevated during the pretrapping period of intense competition around the baited traps than immediately after trapping. Further collections are needed to determine whether fecal cortisol levels differ consistently between anubis and hamadryas, or emerge only in a stressful context. In either case, the difference seems to parallel the species difference in MHPG. 


\section{References}

Asberg, M., Nordstrom, P., \& Traskman-Bendz, L. (1987). Cerebrospinal fluid studies in suicide. Annals of New York Academy of Sciences, 487, 243-255.

Beehner, J. (2004) Female behavior and reproductive success in a hybrid baboon group (Papio hamadryas hamadryas x Papio hamadryas anubis) (Ethiopia). Dissertation Abstracts International A64(12)

Emanuele, E., Brondino, N., Pesenti, S., Re, S., \& Geroldi, D. (2007). Genetic loading on human loving styles. Neuroendocrinology Letters, 2007, 815-821.

Gerald, M., Higley, S., Lussier, I., Westergaard, G., Suomi, S., \& Higley, J. (2002). Variation in reproductive outcomes for captive male rhesus macaques (Macaca mulatta) differing in CSF 5hydroxyindoleacetic acid concentrations. Brain, Behavior and Evolution, 60(2), 117-124.

Jolly, C., \& Phillips-Conroy, J. (2003). Testicular size, mating system, and maturation schedules in wild anubis and hamadryas baboons. International Journal of Primatology, 24, 125-142.

Langlais, P., Bird, E., \& McEntee, W. (2001). Stability of monoamine metabolites in human cerebrospinal fluid. Annals of Neurology, 1982, 48-51.

Manuck, S., Kaplan, J., \& Lotrich, F. (2005). Brain serotonin and aggressive disposition in humans and nonhuman primates. The Biology Of Aggression pp. 65-113. Oxford: Oxford University Press.

Mehlman, P., Higley, J., Faucher, I., Lilly, A., Taub, D., Vickers, J., Suomi, S., \& Linnoila, M. (1995). Correlation of CSF 5-HIAA concentration with sociality and the timing of emigration in free-ranging primates. American Journal of Psychiatry, 152(6), 905-913.

Phillips-Conroy, J., \& Jolly, C. (2004). Male dispersal and philopatry in the Awash baboon hybrid zone. Primate Report, 68, 27-52.

Raleigh, M., Brammer, G., McGuire, M., Pollack, D., \& Yuwiler, A. (1992). Individual differences in basal cisternal cerebrospinal fluid 5-HIAA and HVA in monkeys. The effects of gender, age, physical characteristics, and matrilineal influences. Neuropsychopharmacology, 7, 295-304.

Trefilov, A., Berard, J., Krawczak, M., \& Schmidtke, J. (2000). Natal dispersal in rhesus macaques is related to serotonin transporter gene promoter variation. Behavior Genetics, 30, 295-301. 